\title{
Part-whole relationships in the processing of small visual patterns
}

\author{
NEAL F. JOHNSON, MARILYN TURNER-LYGA, and BARBARA S. PETTEGREW \\ The Ohio State University, Columbus, Ohio
}

\begin{abstract}
Subjects seem to react to a word faster than they react to a letter within a word. One interpretation is that words are processed holistically; another is that all visual stimuli are processed in terms of components, but that more stimulus information is available for use when the targets are words than when they are letters within words. The results of three experiments indicate that the word or pattern-level advantage occurs even when the stimulus information in the two situations is equated, but if the perceptual arrays cannot be unitized (e.g., consonant sequences), a pattern-level advantage does not occur. In addition, the experiments provide substantial evidence to indicate that if letter arrays cannot be unitized, then they are processed on a componentby-component basis, rather than holistically. Finally, the appropriate definition of holistic processing is considered.
\end{abstract}

An experimental paradigm has been employed (Johnson, 1975) that involves presenting subjects with visual displays that consist of a single word, and, depending upon the condition, the subjects either determine whether the display matches a predesignated target word (word-search task) or determine whether the display contains a predesignated target letter (letter-search task). The results of a variety of experiments using this paradigm indicate that subjects can make word-level decisions faster than they can make decisions regarding component letters (Johnson, 1975, 1977; Marmurek, 1977; Sloboda, 1976, 1977), and this phenomenon has been referred to as the word-priority effect (Sloboda, 1977).

Although the data seem clear in terms of the empirical effect, there is disagreement regarding how the data are most appropriately interpreted. It is tempting to suggest that the data indicate that words are processed holistically, in that word-level information does seem to be available before that of components, but other interpretations have been offered that do not require that assumption. For example, Sloboda (1977) suggested that the word-priority effect may stem from comparison-stage events. In particular, if the task of comparing a target letter to the letters within a word is more difficult than the task of comparing a target word to a displayed word (e.g., more comparisons are needed in the letter-search task), then that difference in comparison-stage difficulty could explain the word-priority effect without appealing to any idea of holistic encoding. In order to illustrate his point, Sloboda demonstrated that with increases in the number of letter-to-letter comparisons needed in the letter-search task, there also was an increased advantage for the word-

Requests for reprints should be sent to the first author at the Department of Psychology, 404C West 17th, The Ohio State University, Columbus, $\mathrm{OH} 43210$. search task over the letter-search task (i.e., the wordpriority effect got larger).

Although Sloboda is clearly correct in noting that the magnitude of the word-priority effect is influenced by the number of comparisons needed in the letter-search task, there is evidence that the entire word-priority effect cannot be explained in those terms. For example, in the third experiment of Johnson's (1975) study, a control was included to handle that specific problem in that the subjects in the control condition were to confine their search to only the first letter position. Under those circumstances the number of comparisons (i.e., one) needed for the letter-search task would be the same as for the wordsearch task. The data indicate that when such a control is included to equate the number of comparisons (Johnson, 1975; Johnson \& Marmurek, 1978), the magnitude of the word-priority effect is reduced, but it is still present, indicating a residual effect that cannot be explained in terms of the type of comparison-stage event suggested by Sloboda.

A somewhat more difficult issue was raised by Henderson (1975). He noted that in most studies that demonstrated a word-priority effect, there were few if any overlapping letters between the targets and foils (i.e., the displays that do not match the predesignated target). If subjects did process words on a letter-by-letter basis, the lack of letter overlap between targets and foils would allow subjects to make word-level decisions on the basis of the first letter that was processed, resulting in a faster response than if the subject had to seek out a specific letter within the displayed word.

A view similar to Henderson's has been suggested by Massaro and Klitzke (1977). They assumed that subjects extract features from all letter positions within words in parallel, and if there is little or no overlap in letters between targets and foils, the subject has enough information to make a word-level decision (i.e., to decide whether 
the display matches the target) the moment he or she extracts at least one feature from each letter position. On the other hand, if the perceiver must make a decision regarding a letter within a word, the fact that any one letter shares so many features with other letters requires the subject to extract many features from each letter before a decision can be made.

The Henderson (1975) and Massaro and Klitzke (1977) views regarding the word-priority effect are slightly different, but for the present study, the most important aspect of these and similar models (e.g., Chambers \& Forster, 1975 ) is their common assumption that subjects are able to truncate the process of word identification and make word-level decisions on the basis of component-level representations or encodings. This idea can be referred to as the truncated-processing hypothesis, which has two critical assumptions: (1) that the initial cognitive representation of any visual display is in terms of specific encodings of its components (e.g., letters or features, if the display is a word), and (2) that subjects can decide whether a display as a whole matches a predesignated target pattern on the basis of whether the display's components are consistent with the target.

Quite obviously, the degree of truncation can vary all the way from the subjects' comparing every component of the display with every component of the target, to their making a decision on the basis of a single component-tocomponent comparison. However, the general case should be that the moment at which any component inconsistent with the target is encoded, the subject should immediately terminate the encoding process and respond "no," but in the case of a match, depending on the subject's criterion, more extensive encoding may have to occur before the subject can respond "yes." In both cases, however, the subject should not have to do any patternlevel encoding before making a matchmismatch decision, and it is assumed that there are viable component-level representations that can be used as a basis for pattern-level decisions.

The alternative view assumes that truncated processing is not a general characteristic of the way perceivers deal with words, and that perceivers do not have the requisite component encodings available in a form that allows them to truncate processing. That is, this alternative view assumes that at no time prior to a perceiver's having a usable cognitive encoding of a presented word, does he or she have an analogous encoding of any component that can be used as a basis for making a decision.

This latter view has been incorporated within the pattern-unit model of word processing (Johnson, 1975, 1977,1981 ) in that it follows from several of that model's assumptions. Specifically, that model assumes that whenever a small visual pattern is presented, the subject's initial attempt is to encode it into memory in terms of a holistic pattern-level encoding, regardless of the nature of the display, and that component-level processing occurs only after some fixed number of attempts at patternlevel encoding have failed. The model further assumes that those initial attempts would be successful either if the subjects have a prelearned code that can be assigned to the display, or if they know a rule system that can be used for constructing or generating such a code. Under those circumstances, the display always would be processed into memory as a unit, and the perceiver never would have a component-level representation.

An example of this latter possibility would be a word, in which case the subject could use his or her knowledge of the orthographic structure of the language to construct a word-level code for representing the display in memory, and there would be no usable intervening cognitive representations of either single features or single letters. On the other hand, if the presented display consisted of an unfamiliar consonant sequence, the initial attempts to assign a unitary pattern-level encoding would fail, and it then would be necessary for the subjects to parse the display into components and process them into memory on an element-by-element basis.

In addition, it is important to note that only through the failure to successfully encode the display holistically can the perceiver know that the display cannot be handled in that manner (i.e., initial attempts to process small patterns are always holistic). Given that is the case, the perceiver's initial attempts to encode the display should not be determined in any way by characteristics of the display itself. That is, regardless of whether the display is a unitizable word or a nonunitizable consonant sequence, the same initial attempt at holistic encoding would occur (albeit it would be unsuccessful in the case of the consonants).

In addition, this latter point would suggest that relative to making a decision regarding a letter in isolation, there should be a delay in the decision concerning a letter in the context of other letters, regardless of whether the target and context form a word or simply an array of consonants. If they form a word, the delay would result from the initial word-level encoding, as well as from the subsequent decoding of the word code into letter codes, whereas if they form a consonant array, the delay should stem from the initial unsuccessful attempt at pattern-level encoding, and Sloboda (1976) has reported data supporting these expectations.

The focus of the experiments presented here was on the conditions under which truncated processing can occur, and the empirical consequences of such processing. The truncated processing of a displayed pattern is defined as a subject's initial attempt to encode a pattern's components, with the subject then making a pattern-level match-mismatch decision on the basis of the consistency between the set of encoded components and those of the target. Although the general case for truncated processing in this word-detection task is the making of a patternlevel decision based only on component-level encodings, with no involvement of any pattern-level encoding, the degree of truncation can range from making a decision on the basis of the first letter encoded to delaying the decision until all letters are encoded. In the present experiments, the specific questions explored were whether truncated processing can account for the word-priority effect 
(Experiment 1), and whether truncated processing is a general characteristic of all pattern encoding, or whether it is unique to a few well-defined conditions (Experiments 2 and 3 ).

In the context of the latter question, although these experiments were not designed to compare any one specific model with any other, the particular issue examined in Experiments 2 and 3 was the extent to which the patternunit model provided an adequate specification of the conditions under which truncated processing does and does not occur. In particular, if perceivers have acquired either a prelearned code or a rule system that can be used to generate an integrated representation of the display, then truncated processing should not be possible. However, if subjects have no way of providing such an integrated representation, then it should be possible to truncate processing.

\section{EXPERIMENT 1}

The general task used in Experiment 1 was to present subjects with visual displays that consisted of single words typed in uppercase letters, and the subjects' specific task was to determine whether the display matched a predesignated target word (word-search task) or whether it contained a predesignated target letter (letter-search task). In addition, the "no" or foil items for the word-search task always differed from the predesignated target word by only a single letter (e.g., NEST vs. NEXT or LAND vs. SAND), and when those displays were used in the lettersearch task, it was always the specific differentiating letter that was used as the target or foil. By using stimulus materials of this sort, one can ensure that the stimulus information used in the two tasks is exactly the same. Although Sloboda (1977) previously reported evidence of an advantage for word-level decisions under these circumstances, the issue seemed sufficiently important to warrant beginning the present series of experiments with a replication of that basic effect.

In addition to varying the type of search task, our original intent was to vary the similarity of the critical letter to its corresponding letter in foil displays. We sought to discover the extent to which the confusion effects obtained when subjects make decisions regarding single letters (Yaworsky \& Johnson, 1981) might be reduced or eliminated when the task required a word-level decision.

Unfortunately, it became apparent as the materials were being constructed that it would be impossible to even approximate the constraints required by the counterbalancing and controls that were needed to make the similarity manipulation meaningful. For example, not only are there serial-position effects in a task such as this, but those effects might interact with the type of similarity relationship, and that interaction would require that these two factors be counterbalanced. In addition, in the letter-search task, there would be no way of isolating the letter in the foil items that corresponds to the critical letter. Therefore, it would have been necessary that all the letters in those words be dissimilar to the critical letter in order to avoid uncontrolled confusions.

What makes that problem particularly difficult is that it would not exist for the word-search task, because the single critical letter distinguishing target words and their corresponding foil displays is always readily apparent, as it is the only differentiating item. Therefore, in order to examine the issue of similarity, it would have been necessary to use only words in which the critical letter was physically dissimilar to all the other letters, and for which it would be possible to counterbalance similarity and serial position. Unfortunately, it was impossible to obtain a set of words that would meet all of these conditions.

Nevertheless, we decided to try to control for overall target-to-foil similarity by having half of the foil items have critical letters that were similar to the corresponding display letter and having them different for the other half. However, there was no attempt to control for the similarity of the critical letter to the other letters in the word. Therefore, by including this manipulation, we ensured that a range of similarity relationships was included in the experiment, but that any specific comparison between levels of similarity would not be particularly meaningful.

\section{Method}

All of the displays were four-letter words, and they were presented to the subjects in blocks of 16 , with a brief rest between blocks. Each display consisted of a single word, typed in 10-point uppercase letters (IBM Orator) with a 12-point typewriter. A single space was typed between each letter to avoid crowding the letters, resulting in displays that occupied a visual angle of about $1^{\circ}$. From the subject's 31 -in. viewing distance, the letters appeared appropriately spaced.

Word frequency was not controlled in this experiment, because prior data (Johnson, 1977, 1981) indicated that even extreme variations in frequency have no influence in this word-detection task. That, of course, is as it should be, because providing the subject with the predesignated target in advance should act as a prime for that item, which would eliminate any effect of cultural frequency.

Within each block of 16 displays, 8 displays conformed to the predesignated target (yes items) and 8 were foils (no items). For both the 8 foils and 8 targets, the critical letter (or corresponding foil letter) appeared in each of the four letter positions equally often. Additionally, in 4 of the 8 foils, the critical letter was similar to the corresponding letter in the display, and in 4 , those letters were dissimilar.

The dimension of similarity was defined by dividing the letters of the alphabet into three classes, in which similar items were letters from the same class and dissimilar items were letters from different classes. The three classes were: (1) letters composed of only vertical and horizontal lines, (2) letters that contained a diagonal (except $\mathrm{R}$ was categorized as a curve and was never compared to a diagonal), and (3) letters that contained a curve. In the case of a dissimilar relationship, a distinction between the first two classes was never used, because prior data (Gibson \& Levin, 1976) indicated that these two classes are confused a great deal (e.g., in reality, they actually may be two subclasses of one larger class).

There were 192 displays, divided into 12 blocks of 16 items each. For the 96 displays in which the target item did appear (i.e., the yes items), the letters used as the critical letters, and the frequency with which they were used as critical letters, were the following: A. $16 ; \mathrm{B}, 4 ; \mathrm{C}, 2$; D, 5; E, 10; F, 1; G, 2; H, 3; I, 4; J, 1; K, 4; L. $3 ; \mathrm{M}, 4 ; \mathrm{N}, 6 ; \mathrm{O}, 5 ; \mathrm{P}, 6 ; \mathrm{R}, 3 ; \mathrm{S}, 7 ; \mathrm{T}, 7 ; \mathrm{V}, 2 ; \mathrm{W}, 1$. The critical or target letters and their respective frequencies for foil displays were: A, $2 ; \mathrm{B}, 2 ; \mathrm{C}, 2 ; \mathrm{D}, 7 ; \mathrm{E}, 7 ; \mathrm{F}, 3 ; \mathrm{G}, 2 ; \mathrm{H}, 3 ; \mathrm{I}, 7$; 
K, 5; L, 7; M, 6; N, 4; O, 4; P, 9; R, 10; S, 5; T, 2; U, 5; V, 4: and the letters and their frequencies from the foil displays that corresponded to the critical letters were the following: A, 2: B, 6; C, 4; D, 8; E, 8; G, 4; H, 6; I, 4; K, 6; L, 6: M, 6; N, 8; O, 2: P, 2: R, 4; S, 6; T, 6; U, 4; W, 2; Z, 2 .

For six blocks of 16 displays, the subjects were given word targets, and for the other six blocks, the targets were single letters. Half the subjects performed the word-search task first, followed by the letter-search task, whereas the reverse was true for the other half of the subjects. In addition, the displays in any one block were used for the word-search task for half the subjects and for the lettersearch task for the other half of the subjects, and that counterbalancing was crossed with the counterbalancing of the order in which the tasks were presented to the subjects. Finally, the order of the items within a block was randomized after every 4 subjects.

The switch that triggered the onset of the displays also started a timer that was turned off when the subject made his or her response. The subject responded "yes" or "no" by pressing one of two buttons, and was given the option as to which hand to use for each response, but the subject was not allowed to switch hands once the testing began.

The displays were presented to the subjects at a rate of about one every $10 \mathrm{sec}$. The subjects viewed a predisplay fixation point, and immediately prior to the onset of each display, the experimenter stated the name of the target the subject was to use for that display. In the case of word targets, the words were pronounced to the subject, but not spelled. The experimenter then said "ready," and that was followed after about 500 msec by the onset of the display, which remained in view for $500 \mathrm{msec}$. The display apparatus was a twochannel tachistoscope, Scientific Prototype Model 800E. The subjects were 24 students who volunteered as part of a course option.

\section{Results}

The latency and error data are presented in Table 1. The overall error rate was $4 \%$. An analysis of that data indicated that none of the experimental manipulations had a reliable effect on errors $[F<1$ for both main effects, and for the interaction, $F(1,23)=2.43, p>.05$ ]. In addition, the correlation between the error and latency data given in Table 1 is +.35 . In general, then, the data do not indicate any evidence of a significant speed-accuracy trade-off.

The latency results were subjected to a similar analysis, and the latency advantage for the word-search task was reliable $[F(1,23)=21.49, p<.001]$, as were the advantage for "yes" responses $[F(1,23)=288.30$, $p<.001]$ and the interaction $[F(1,23)=24.87$, $p<.001]$. The interaction indicated that the effect of the type of search task was considerably larger for the "yes"

Table 1

The Latencies (in Milliseconds) and Mean Number of Errors for Each Condition in Experiment 1

\begin{tabular}{llcc} 
& \multicolumn{2}{c}{ Response Type } & \\
\cline { 2 - 3 } Search Task & Yes & No & Mean \\
\hline Letter & 589 & 647 & \\
$\quad$ Latency & 2.42 & 1.88 & 618 \\
$\quad$ Errors & & & 2.15 \\
Word & 509 & 623 & \\
$\quad$ Latency & 1.79 & 2.33 & 566 \\
$\quad$ Errors & & & 2.06 \\
Mean & 549 & 635 & \\
$\quad$ Latency & 2.11 & 2.11 & 592 \\
$\quad$ Errors & & & 2.11 \\
\hline
\end{tabular}

responses than for the "no" responses, but even the difference for the latter was reliable at the .05 level (one tail) $[t(23)=1.83]$.

Finally, for the foil items, there did appear to be a small overall confusion effect based on target-to-foil similarity $(643 \mathrm{msec}$ vs. $628 \mathrm{msec})[t(23)=1.98, p<.05$ (one tail)], replicating prior studies, but the magnitude of that effect was literally identical for the two search conditions, offering no evidence that the effect might be differential. However, as noted above, even if there had been a differential effect, the lack of appropriate controls would have precluded any meaningful interpretation of those data.

\section{Discussion}

The major purpose of Experiment 1 was to determine whether the latency advantage for word-level decisions over decisions about letters within words (i.e., the wordpriority effect) was dependent upon the type of severely truncated processing described by Henderson (1975) and Massaro and Klitzke (1977). These investigators proposed that subjects identify a word by first attempting to encode the letters or features, but then terminate the componentlevel processing when enough of the display's component codes are available to determine whether they match those of the predesignated target. If targets and foils share few, if any, letters, the word-level decision can be based on the first letter or two encoded, regardless of which letters they might be. However, if the task is to make a decision as to whether the display contains a particular letter, the subjects can react only when that letter has been encoded, and it may not be one of the first to be encoded.

Consistent with Sloboda's (1977) study, the results of this experiment indicated that such a strategy effect cannot provide a complete account of the word-priority effect. That is, the subjects were faster in the word-search task than in the letter-search task, even when target-tofoil similarity was controlled such that subjects could not truncate processing when engaged in the word-search task.

Although the word-priority effect was obtained for both the "yes" and the "no" responses under these conditions, a slight complication in the results was the fact that the difference between the letter-search and word-search tasks did interact with response type. However, that task $x$ response type interaction can be explained in the context of the pattern-unit model by noting that it occurred because of a smaller difference between the "yes" and "no" responses for the letter-search task than for the word-search task. Following Krueger (1970), it may be reasonable to assume that a "yes" response is made on the basis of a holistic match between the target and the stimulus, whereas in the case of a mismatch, the subject must then make a subsequent component-level double check before responding. If this assumption is correct, it would be expected that "yes" responses would be faster than "no" responses, and the data from many reaction-time studies indicate that that is generally the case.

However, it also is the case that the latency for "no" responses should be some function of the difficulty sub- 
jects have in making the component-level double check. If the subject's target was a word, the "yes" response could occur immediately after the holistic word-level match, but if the comparison resulted in a mismatch, the subject then would have to decode the word-level code into component letters before making the component-level double check. That extra decoding, plus one or more component-level comparisons, would delay the "no" response.

The situation would be somewhat different if subjects were to respond to a letter within the word. Under those conditions, the decoding of the word-level code into components would have occurred before any comparison process began, which would explain why the "yes" response is delayed relative to the "yes" response for a word-level decision. However, in the event of a mismatch at that point, the subjects again would make a double check, but in the letter-search task that would not have to be preceded by any decoding steps, as would be the case for the word-search task. That is, those decoding steps already would have occurred before the initial comparison process started, and that should result in a smaller difference between "yes" and "no" response latencies for the letter-search task than for the word-search task.

\section{EXPERIMENT 2}

Because these data, as well as data previously reported by Sloboda (1977), indicate that the word-priority effect is not dependent upon subjects' severely truncating their word processing, the next question is whether truncated processing can ever occur, and if so, under what circumstances and to what degree it can occur. Again, truncated processing refers to the hypothesis that subjects respond to a word-level pattern by first attempting to encode the word's letters or features, but then terminating that component-level processing and making a pattern-level decision on the basis of whether the initially encoded subset of components was consistent with those contained in the target.

In terms of the pattern-unit model, the expectation from the foregoing considerations is that if subjects do have a prelearned code for a displayed pattern (e.g., a singleletter code), or they know a rule system that can be used for constructing such a code (e.g., a word code), then it would not be possible for them to truncate their patternlevel processing of that item. That expectation means that only pattern-level characteristics, and not component-level characteristics, should influence the processing of the display.

On the other hand, if the subject has no way of providing an adequate unitary encoding for the pattern as a whole (e.g., a consonant sequence), the initial attempt to encode it holistically should fail, and the subject would have to process it on a component-by-component basis. However, such component-by-component encoding would allow for the possibility of truncated processing (i.e., the subject could make a pattern-level decision on the basis of a match or mismatch of component-level encodings).

Given this general set of expectations, the next question concerns the way these effects might be realized in the context of the specific word-detection paradigm employed in these experiments. First, however, it must be noted that for letter displays, the expectations are somewhat different depending upon whether the targets and foils share letters, and whether in the letter-search task the perceivers know the specific location of the critical letter (i.e., the target or foil). This would be particularly true in situations where truncated processing is possible. To simplify matters, and for reasons that will become apparent, for the next two experiments, we always used displays in which targets and foils never shared letters, but the subjects were not informed of that fact. ${ }^{1}$ In addition, in the letter-search task, the critical letter always appeared only in the first letter position, and the subjects were in formed of that fact. Therefore, the following set of expectations is based on the assumption that the task has these characteristics.

In the context of this task, the pattern-unit model expects that if the displays are pronounceable, they should be processed holistically. If that were the case, then a pattern-level decision should be made faster than a component-level decision, because the latter would require the additional step of decoding the pattern into components. In addition, the "yes" responses should be faster than the "no" responses, because the "no" responses would require the double check that was described earlier. Finally, because the decisions in both tasks (i.e., patternlevel and component-level decisions) would be based on a single comparison of two holistic representations, there should be no effect of the size of the display on the response latency.

The situation would be somewhat different if the displays were consonants, and subjects could not provide a unitary encoding. In that case, the model would expect the display to be processed on a component-by-component basis, allowing for the possibility of truncated processing. First, when subjects are to match a target letter to the first letter of the display, they need make only a single letter-to-letter comparison, which should be faster than matching the entire display to a predesignated target sequence. In this latter situation, the task would require subjects to make multiple comparisons.

In addition, for the pattern-level decision, if multiple comparisons are needed for the "yes" responses, there should be an effect of the size of the display. That, of course, would depend upon the criterion of certainty adopted by the subject, and the task would allow the subjects to respond "yes" on the basis of having encoded a single compatible letter (i.e., targets and foils never share letters), but prior data indicates that subjects are unable to capitalize on that fact in this situation (see Note 1). Consequently, display size should influence the "yes" responses when the displays are consonant arrays. 
However, in the case of a mismatch or no item for the task, the fact that targets and foils never shared letters would mean that the first letter-level comparison would give the subjects the information that they need to respond "no," and that should result in "no" responses being faster than "yes" responses, with the effect of display size being confined to the latter. On the other hand, if subjects were to compare a target letter to the first letter of a display, only a single comparison would be needed for both yes and no items, but the double check of the no item should give the standard latency advantage for the "yes" responses.

Therefore, when truncated processing is possible (e.g., consonant arrays), if targets and foils never share letters, but the subject is not informed of that fact, and the lettersearch task is confined to a single letter position, then (1) component-level decisions should be faster than pattern-level decisions (i.e., the reverse of what should occur for word displays); (2) "no" responses should be faster than "yes" responses for pattern-level decisions (again, the opposite of what should occur for word displays); (3) the standard latency advantage for "yes" responses should be obtained for component-level decisions; and (4) whereas a pattern-level "yes" response should be influenced by display size (that would not occur if the display was a word), that should not be true for the pattern-level "no" responses, or for either response when subjects make a component-level decision.

Turning now to the specifics for Experiment 2, the subjects always made pattern-level decisions to determine whether an entire display matched a predesignated target. The targets consisted of strings of either three or four letters. In addition, there were three types of display, either consonant strings, for which truncated processing would be possible, or words or pronounceable nonwords for which truncated processing should not be possible. ${ }^{2}$ Again, truncated processing is defined as making a wordlevel decision on the basis of one or more initially encoded letters or features. If those components are consistent with the predesignated target, the subject responds "yes," but the subject responds "no" if there is any mismatch. That is, the pattern-level match-mismatch decision is based on one or more comparisons of componentlevel encodings.

We predicted that when truncated processing cannot occur (i.e., the displays are words and pronounceable nonwords), "yes" responses should be faster than "no" responses, but there should be no main effects for either length or type of display. Also, there would be no basis for expecting any interactions among these variables. For the consonant strings, on the other hand, "no" responses should be faster than "yes" responses and there should be an overall effect of length, but the effect should be confined to the "yes" responses, yielding a length $\times$ response type interaction.

In general, these predictions can be summarized by noting that the reaction to the consonant arrays should be slower than to the other two types of display, the effect of length should be confined to the "yes" responses for the consonant displays, and although "yes" responses should be faster than "no" responses for the two types of pronounceable display, the reverse should be true for the consonant displays. In short, if subjects truncate processing under the conditions of these experiments, then "no" responses should be faster than "yes" responses, and there should be an effect of display size confined to the "yes" responses.

\section{Method}

The procedures for Experiment 2 were similar to those used in Experiment 1. As in Experiment 1, the displays were typed in 10point Orator type on a 12-point typewriter with a single space between each letter. Each subject received six blocks of 24 displays, with one block representing each combination of the two lengths (three and four) and three types of display (words, pronounceable nonwords, and consonant strings), and the subjects were informed regarding the characteristics of the items within a block before it was presented. In addition, for half of a block's displays, the displays conformed to the predesignated target (which was different for each display), and for half, there was a mismatch between the target and display (foils). For any foil display, the only restrictions on its predesignated target were that it not share any letters with the display, and that it not conform to any other display presented to the subject, but the subjects were not informed of these restrictions. Finally, an equal number of subjects had each display as a target item and as a foil item, and the order of the items within a block was randomized after every 4 subjects.

To make this experiment as comparable as possible to the earlier studies, we allowed the subject to select the hand they wished to use for each response. As usual, essentially all the subjects chose the right-yes and left-no combination (even left-handed subjects). However, the subjects were required to make that choice before they were informed regarding the nature of the displays, and they were not allowed to change their decisions during the experiment. Because the reaction time should be shorter for the preferred hand, one might expect an overall advantage for "yes" responses; however, the critical issue in the experiment was whether the effect of response type interacts with the effects of display type and length, and although allowing the subjects to choose the hand they wished to use for each response would contaminate the main effect, it should not have any biasing effect on those interactions.

The four-letter words and nonwords conformed to either a CCVC or a CVCC letter pattern, and the three-letter words and nonwords had a CVC pattern. The words had frequency ratings of $A$ and AA (Thorndike \& Lorge, 1944), and the pronounceability of the nonwords was evaluated by two judges, who had to agree that an item was pronounceable before it was included in the set. The only restriction on the consonant strings was that they not be a generally familiar sequence, such as commonly recognized initials. Again, the criterion was the agreement of the two judges. The visual angles of the three- and four-letter displays were $.78^{\circ}$ and $1.09^{\circ}$, respectively.

To increase the sample of items in the experiment, we used five different sets of materials, a different set for the subjects in each of five different counterbalancing cycles (described below). Therefore, each combination of length, response type, and type of display was represented by 120 different items in the experiment.

Immediately before each display was presented, the target for that item was given to the subject. In the case of the consonant strings, the target was spelled for the subject, but for the pronounceable displays, the target was first pronounced and then spelled. The experimenter then said "ready" and the display appeared about $500 \mathrm{msec}$ later.

The blocks of displays for the two lengths for any type of display were presented adjacently, and the six possible orders in which 
the three types of display could occur were used equally often. For any display type, the subjects always received all of their threeletter displays immediately before their four-letter displays, or vice versa; that counterbalancing, combined with the six orders in which the conditions (display type) could be presented and with the two levels of a display's function (i.e., target or foil), resulted in counterbalancing cycles of 24 subjects. Five such cycles were used, resulting in a sample of 120 subjects. The subjects were students who volunteered as part of a course option.

\section{Results}

The error data are presented in Table 2. The overall error rate was $3 \%$, and an analysis of those data indicated a reliable effect of both length $[F(1,119)=9.00$, $p<.01]$ and type of response ("yes" vs. "no") $[F(1,119)=4.51, p<.05]$, as well as a length $\times$ display type interaction $[F(2,238)=5.51, p<.05]$, but none of the other effects was significant. These results suggest that possible speed-accuracy trade-off effects might cloud the interpretation of any overall reaction time advantage for "yes" responses and for short displays, but neither of these were critical issues within this experiment.

However, not only were these error differences quite small, but the most critical latency data in the study are reflected in the length $x$ response type interaction and the length $\times$ response type $x$ display type interaction, and none of these effects was reliable for the error data, with $F<1$ in all cases. In addition, although the error data did yield a reliable length $\times$ display type interaction, the correlation between errors and latency for the six means involved in that interaction was +.13 . Furthermore, an overall correlation was computed between errors and latency, with the 12 conditions involved in this experiment being the sampling units, and the correlation was only -.07 , which also suggests that there may not

Table 2

The Latencies (in Milliseconds) and Mean Number of Errors per Subject for Each Condition in Experiment 2

\begin{tabular}{|c|c|c|c|c|}
\hline \multirow[b]{2}{*}{ Display Size } & \multicolumn{3}{|c|}{ Display Type } & \multirow[b]{2}{*}{ Mean } \\
\hline & Consonants & $\begin{array}{c}\text { Pronounceable } \\
\text { Nonwords }\end{array}$ & Words & \\
\hline \multicolumn{5}{|c|}{ Three-Letter Words } \\
\hline \multicolumn{5}{|l|}{ Yes } \\
\hline Latency & 532 & 478 & 447 & 486 \\
\hline Errors & .53 & .47 & .37 & \\
\hline \multicolumn{5}{|l|}{ No } \\
\hline Latency & 522 & 487 & 480 & 496 \\
\hline Errors & .41 & .35 & .31 & \\
\hline Mean Latency & 527 & 483 & 464 & 491 \\
\hline \multicolumn{5}{|c|}{ Four-Letter Words } \\
\hline \multicolumn{5}{|l|}{ Yes } \\
\hline Latency & 587 & 489 & 449 & 508 \\
\hline Errors & .36 & .24 & .42 & \\
\hline \multicolumn{5}{|l|}{ No } \\
\hline Latency & 535 & 500 & 469 & 501 \\
\hline Errors & .33 & .18 & .33 & \\
\hline Mean Latency & 561 & 494 & 459 & 505 \\
\hline Grand Mean & 544 & 488 & 461 & 498 \\
\hline
\end{tabular}

be much overall speed-accuracy trade-off for tasks in this experiment.

The latency data are presented in Table 2 . An inspection of the table indicates that the data are in rather close accord with expectations. There was a main effect for display type (bottom row of Table 2) $[F(2,230)=27.57$, $p<.001]$, and the major factor was the expected very slow responses to the consonant displays, as compared to responses to the two types of pronounceable displays. In addition, although there was a main effect for length $[F(1,115)=5.90, p<.05]$, the effect seemed to be confined to the task involving consonant displays, and that point is supported by a reliable interaction between display type and length $[F(2,230)=7.19, p<.001]$.

The main effect for response type ("yes" vs. "no") was not reliable ( $497 \mathrm{msec}$ vs. $498 \mathrm{msec})(F<1)$, but that effect interacted with both length $[F(1,115)=10.70$, $p<.01]$ and display type $[F(2,230)=7.19, p<.01]$. That is, the effect of length seemed to be confined to the "yes" responses, and whereas the usual latency advantage for "yes" responses was obtained for the pronounceable displays, the anticipated reversal of the effect occurred when the displays were consonants. Finally, in that the length effect for "yes" responses was expected to occur for only the consonant displays, the interaction between response type, display type, and length apparent in Table 2 also was anticipated $[F(2,230)=4.82$, $p<.01]$.

The particular hypothesis involved in this experiment can be examined in terms of specific comparisons among the conditions. First, for the consonant strings, for which truncated processing should be possible, the anticipated effect of length was obtained $[F(1,115)=10.94$, $p<.01]$, the latency advantage for the "no" responses was reliable $[F(1,115)=18.45, p<.01]$, and the interaction indicated that the length effect was more or less confined to the "yes" responses $[F(1,115)=11.17$, $p<.01]$. In addition, the length effect for the consonant strings was significantly greater (i.e., the interactions) than that obtained for either the pronounceable nonwords $[F(1,115)=42.7, p<.05]$ or the words $[F(1,115)=$ $12.32, p<.01]$. Finally, the overall latency for the consonant strings was longer than that obtained for either the pronounceable nonwords $[F(1,115)=22.58, p<.01]$ or the words $[F(1,115)=46.77, p<.01]$.

A comparison between the words and the pronounceable nonwords offered no support for an effect of length on reaction time $(F<1)$ and there was no interaction $[F(1,115)=3.51, p<.05]$. In addition, although the expected advantage for the "yes" responses was obtained $[F(1,115)=23.62, p<.01]$, it did interact with display type $[F(1,115)=6.53, p<.05]$, with the reliability of the advantage for "yes" responses being rather borderline for the pronounceable nonwords $[t(114)=1.90$, $p<.05$ (one tail)]. Finally, another unanticipated result was a reliable advantage for the words over the nonwords $[F(1,115)=7.12, p<.05]$. 


\section{Discussion}

Whereas Experiment 1 demonstrated that the wordpriority effect is not dependent upon the type of truncated processing described by Henderson (1975) (i.e., the effect was obtained in conditions under which subjects could not truncate processing), the focus of Experiment 2 was on whether it was possible to truncate processing when the displays and targets consisted of items that can be integrated, such as words. This somewhat stronger hypothesis is an implication of the pattern-unit model, and it would be supposed that truncated processing occurs only when the subjects are unable to provide a unitary representation for a display. That is, if subjects do have an available pattern-level code, then they should not be able to make a pattern-level decision on the basis of an incomplete set of component-level encodings.

In these terms, the results of the experiment are quite straightforward. The fact that targets and foils did not share letters (albeit the subjects did not know that) should have allowed subjects to make a "no" decision regarding a foil on the basis of a single letter-to-letter comparison if they had truncated processing, whereas multiple comparisons should have been needed for a "yes" decision. However, in the case of displays which the model expected to be processed holistically (i.e., the pronounceable items), the data indicated no evidence of truncated processing. The "yes" responses were faster than the "no" responses for both words and pronounceable nonwords, and there was no evidence of any length effect for either response type.

On the other hand, for consonant arrays, for which the model expected processing to be truncated, the data clearly suggested that such an effect did occur. The "no" responses were faster than "yes"' responses, and the expected length effect for the "yes" responses was obtained in that situation. In addition, consistent with the previously described data (Note 1), the length effect for the "yes" responses indicates that subjects were unable to capitalize on the fact that targets and foils did not share letters, and that probably occurred because the subjects failed to notice the lack of similarity.

Finally, there were two outcomes that were unexpected. The first was the fact that subjects responded to pronounceable nonwords more slowly than to words, and the second was the fact that the difference between "yes" and "no" responses was smaller for the nonwords than for the words.

One simple explanation that could handle both these effects is that the pronounceable nonwords may not have been as orthographically regular (i.e., rule conforming) as were the words. For example, it may have been that on some trials, the first attempt to assign a unitary representation to a pronounceable nonword was unsuccessful, and that although subjects may have eventually succeeded in handling the display holistically, the initial unsuccessful attempts delayed the response. In addition, there may have been some trials on which subjects never succeeded in assigning a unitary representation to the dis- play, and not only would those trials increase the overall latency for the nonwords, but "no" responses should be faster than "yes" responses, which would tend to reduce the overall latency advantage for "yes" over "no."

\section{EXPERIMENT 3}

In the final experiment, as in the first, the focus was on part-whole relationships as they relate to the issue of truncated processing. In Experiment 1, the similarity relationship between targets and foils was carefully controlled to severely reduce the amount of truncated processing that was possible, and yet the subjects still made pattern-level decisions more rapidly than they made component-level decisions (i.e., the word-priority effect was obtained). Experiment 3 was designed to examine these part-whole relationships when the possibility of truncated processing was maximized. That is, the displays were consonant arrays, and the targets and foils never shared any letters, but as in Experiment 2, the subjects were not informed regarding the target-foil similarity relationship. In addition, in the letter-search task, the subjects were to confine their search to the first letter position.

Given that the results of Experiment 2 did indicate that subjects can and do truncate processing under the circumstances just described, the specific issue involved in Experiment 3 was whether that strategy is sufficient to yield a latency advantage for pattern-level decisions over component-level decisions, as Henderson (1975) suggested might be the case, or whether the reverse effect occurs, as suggested by the pattern-unit model. That is, the model expects that only a single comparison would be needed for both "yes" and "no" responses for the component-level decision, and for the "no" responses for the pattern-level decision, whereas multiple comparisons would be needed for a pattern-level "yes" response. That should result in (1) an overall latency advantage for component-level decisions (i.e., a reverse word-priority effect); (2) the "no" responses should be faster than the "yes" responses for the pattern-level decision; and (3) the latency for the "yes" responses should be an increasing function of display size.

In addition to the foregoing expectations, however, the model expects that truncated processing cannot occur if the task requires a component-level decision. In that subjects would have an available unitary encoding for a single letter (e.g., its name), the encoding of the first letter of the display and its comparison to the target letter should be holistic. If that is the case, the double check needed for " no" responses would result in "yes" responses that are faster than "no" responses, but the latency for neither response type should be influenced by display size.

The displays used in Experiment 3 were arrays of consonants that contained either three or four letters. In the task requiring a pattern-level decision, the predesignated targets were comparable consonant strings of either three or four letters, whereas for the task requiring a component-level decision, the subjects were to determine 
whether the first letter of the displayed array matched a single predesignated target letter.

The predictions from the model are that there should be a main effect for type of task (component-level vs. pattern-level decision), and whereas the main effect of response type should be indeterminant, task and response type should interact such that "yes" responses should be faster than "no" responses for component-level decisions, and the reverse should be true for pattern-level decisions. Finally, there should be a main effect for length, but although it should be confined to the "yes" responses for the pattern-level decisions, there also should be a task $x$ length interaction and a task $x$ length $\times$ response type interaction. In summary, truncated processing should yield "no" responses faster than "yes" responses, and a length effect should be confined to the "yes" responses, whereas holistic processing should yield "yes" responses faster than "no" responses and no length effects.

\section{Method}

The displays were similar to those used for the consonants in Experiment 2 , and they were presented to subjects in eight blocks of 18 displays each, with two blocks representing each of the four combinations of length and type of search task. Within a block, each display began with a different consonant, and no consonant sequence occurred more than once within the experiment.

Half the subjects had the letter-search task before the sequencesearch task, with the reverse being the case for the other half of the subjects. Within each task, half the subjects had the two blocks of three-letter displays before the four-letter displays, with the opposite being true for the other half. Finally, the blocks of displays that were used for each search task for 1 subject were switched for the next, and each display appeared as a yes and a no item for an equal number of subjects. In that way, every display appeared in every condition equally often. All these factors were appropriately counterbalanced, resulting in counterbalancing cycles of 16 subjects.

In all other respects, the procedures and viewing conditions of this experiment were the same as those used in Experiments 1 and 2. The subjects were 32 students who participated as part of a course option.

\section{Results}

The results of the experiment are presented in Table 3. The overall error rate was $3 \%$, and an analysis of those data indicated that neither any of the main effects nor any of the first-order interactions were reliable, with the largest effect being for the length $\times$ response type interaction $[F(1,31)=3.18, p>.05]$, but the length $\times$ task $\times$ response type interaction was significant $[F(1,31)=4.54$, $p<.05]$.

A separate analysis of the error data for each task indicates that the second-order interaction emerged because while neither main effect nor the interaction was reliable for the pattern-level task, with the biggest effect being for length $[F(1,31)=1.03, p>.05]$, there was a significant length $x$ response type interaction for the component-level task $[F(1,31)=6.55, p<.05]$, with $F<1$ for both main effects in that task. However, although there was a reliable interaction for the latter condition, indicating a significant variation in error rate, the correlation between errors and latency was only +.03 ,
Table 3

The Latencies (in Milliseconds) and Mean Number of Errors per Subject for Each Condition in Experiment 3

\begin{tabular}{llll} 
& \multicolumn{2}{c}{ Lengths } & \\
\cline { 2 - 2 } Search Task & 3 & 4 & Mean \\
\hline Yes & Letter & & \\
Latency & 597 & 604 & 601 \\
Errors & .69 & .47 & \\
No & & & \\
Latency & 660 & 662 & 661 \\
Errors & .38 & .81 & \\
Mean Latency & 629 & 633 & 631 \\
& String & & \\
Yes & & & \\
Latency & 652 & 751 & 702 \\
Errors & .47 & .41 & \\
No & & & \\
Latency & 643 & 668 & 655 \\
Errors & .56 & .38 & \\
Mean Latency & 647 & 710 & 678 \\
Grand Mean & 638 & 671 & 655 \\
\hline
\end{tabular}

suggesting that the question of a speed-accuracy tradeoff need not be a point of concern.

An examination of the latency data in Table 3 indicates that the data are substantially in accord with the expectancies. There was a latency advantage for the componentlevel task, which occurred because subjects were very slow on the "yes"' responses for the pattern-level task, but the "no" response latencies were essentially the same for the two tasks. In addition, although there was a length effect, it also appeared to be confined to the "yes" responses in the pattern-level task. Finally, response type did have the expected opposite effects in the two tasks, with "yes" responses being faster in the component-level task, but slower in the pattern-level task.

These observations are supported by an analysis of the data. Although the main effect of response type $(652 \mathrm{msec}$ vs. $658 \mathrm{msec})$ was not significant $(F<1)$, there were reliable effects for both length $[F(1,31)=5.82, p<.05]$ and task $[F(1,31)=4.90, p<.05]$, as well as for the interactions between length and task $[F(1,31)=4.94$, $p<.05]$, length and response type $[F(1,31)=6.52$, $p<.05]$, task and response type $[F(1,31)=31.33$, $p<.001]$, and task, response type, and length $[F(1,31)$ $=7.40, p<.05]$.

Separate analyses for each condition indicated that the only reliable effect for the component-level task was response type $[F(1,31)=28.70, p<.001]$, with $F<1$ for both length and the interaction. For the pattern-level task, on the other hand, there were reliable effects for both length $[F(1,31)=6.37, p<.05]$ and response type $[F(1,31)=9.57, p<.01]$, as well as for the interaction $[F(1,31)=10.66, p<.01]$.

Finally, a separate analysis of the "no" responses indicated no reliable effects, with the largest effect being for length $[F(1,31)=2.59, p>.05]$. For the "yes" responses, there were significant effects for both length $[F(1,31)=7.27, p<.05]$ and task $[F(1,31)=13.99$, 
$p<.01]$, as well as for the interaction $[F(1,31)=7.66$, $p<.01]$.

In summary, (1) there was a significant effect of length that was limited to the "yes" responses for the patternlevel decisions; (2) neither length nor type of task affected the "no" responses; (3) latencies for the pattern-level decisions were longer than for the component-level decisions; and (4) there were the expected opposite effects of response type in the two tasks.

\section{Discussion}

The data indicate that subjects were able to truncate their processing in the task that involved pattern-level decisions. That is, "no" responses were faster than "yes" responses, with the latency for "yes" responses being an increasing function of display size. Both those outcomes seem to suggest that subjects based their pattern-level decisions on comparisons of component-level encodings.

The fact that targets and foils never shared letters should have allowed the subjects to make a "yes" response on the basis of a single letter-to-letter comparison, but the marked effect of display size indicates that that did not happen. That seems to imply that although the processing was truncated when subjects made pattern-level decisions, the degree to which truncation occurred was quite limited. It was the case, of course, that the subjects were not explicitly informed of the target-to-foil relationship, and it is possible that a somewhat more severe truncation of the processing might have occurred had that relationship been described to them. However, the reason for not providing them with that information was to match the conditions under which the word-priority effect was originally obtained, and for which truncated processing has been used as an explanation.

In terms of the word-priority effect, it is clear that even though truncated processing did occur in this experiment, data characteristic of a word-priority effect were not obtained. It took the subjects longer to make their patternlevel decisions than their component-level decisions, which seems to suggest that allowing for the possibility of truncated processing, as well as its occurrence, are not sufficient conditions for the word-priority effect to occur.

Finally, although the subjects did seem able to truncate processing when their task was to make pattern-level decisions, that type of processing did not occur when the target was a single letter and the subjects' task was to make a decision regarding the first letter of the array. To be sure, the consonant array itself was not processed holistically in that situation, but in that the "yes" responses were faster than the "no" responses, it does appear that the individual letters may have been handled in that manner. If the subjects had provided feature-level cognitive encodings prior to the letter-level encoding, it is likely that the time needed to encode enough mismatch information to respond "no" would be substantially less than the time needed to encode enough match information for the subject to be confident of a "yes" response, which would result in a latency advantage for the "no" responses; however, that did not seem to occur in this experiment.

\section{GENERAL DISCUSSION}

The major purpose of the series of experiments presented here was to determine whether the latency advantage for pattern-level decisions over component-level decisions (i.e., the word-priority effect) is a general characteristic of the way subjects deal with any small visual pattern, provided they are able to truncate processing, or whether that effect is unique to situations in which subjects are able to process displays holistically (i.e., displays for which the subject has available a unitary encoding for the item), regardless of whether truncated processing is possible. In addition, although one expects different patterns of data, depending upon whether subjects truncated processing or handled the display holistically, there appears to be an ambiguity both as to whether those differences can be revealed in the context of the specific paradigm employed in these experiments, as well as to the particular conditions under which one or the other type of processing should occur.

\section{Truncated Processing and the Word-Priority Effect}

Regarding the question of whether evidence of truncated processing can be demonstrated in situations such as these, Experiments 2 and 3 indicated that it was very easy to obtain the pattern of data characteristic of truncated processing, provided the displays could not be encoded in a unitary manner. However, when the critical item could be assigned a unitary encoding, the pattern of data was characteristic of holistic processing (i.e., "yes" faster than "no" responses with no length effects), and that seemed to occur regardless of whether the critical item was the entire displayed pattern (Experiment 2) or simply a component letter (Experiment 3 ). It appears, then, that evidence of truncated processing can be obtained in the context of this paradigm, but only when the subject does not have a unitary encoding available that can be assigned to the critical item involved in the comparison.

In terms of the generality of the word-priority effect, the first experiment in this series indicated that the latency advantage for pattern-level decisions can be obtained even when truncated processing cannot occur. In addition, the data from the last experiment indicated that if subjects have no way of encoding the display holistically, the pattern-level decisions are slower than are componentlevel decisions, even when the nature of the task allows for the very severe type of truncated processing described by Henderson (1975). In general, then, the data indicated that whether one obtains a latency advantage for patternlevel decisions over component-level decisions (i.e., a word-priority effect) depends critically on whether the display can be encoded holistically, and the possibility of truncated processing seems to be an irrelevant issue. 


\section{Holistic Processing}

The precise meaning of the concept of holistic processing is clearly open to question. One view that is very appealing, because of both its simplicity and its implications, is that there is no component-level encoding, and that access to pattern-level representations is in some way direct. That rather radical position has been incorporated into the pattern-unit model and, as noted elsewhere (Johnson, 1981), although logic and common sense tell us that such a specific construction of the concept must be wrong (e.g., we do not confuse SHOW and SNOW), the interesting point is that there is very little evidence available to argue against a position of that sort. In fact, the Johnston and McClelland (1980) study may provide the only data for which that position has clear problems (also see Johnston, 1981).

These investigators demonstrated that whereas a visual mask consisting of complete letters reduces a subject's ability to report the letters from both word and nonword arrays, and to an approximately equal degree, a mask containing no complete letters, but just letter features, had a substantially smaller disrupting effect on the report of letters from words than from nonwords. If one goes directly from some form of preliminary visual representation to a pattern-level code, without going through any intermediate levels of representation, it is difficult to explain why the level of representation contained in the mask should differentially influence the availability of components of words but not of nonwords.

However, despite these considerations, there are alternative constructions of holistic processing which not only can cope with extant data, but seem more in accord with what our common sense tells us must be the case. In particular, a conservative definition of the concept can be limited to a simple statement that pattern-level representations are available to the execution of cognitive operations prior to representations of component-level information, without making any specification as to the basis for such differential availability.

Given this more limited definition of the concept, there are a number of models of word recognition that can handle the phenomenon, provided some varying number of other assumptions are included. The Chambers and Forster (1975) race model can cope with the effect if it is assumed that word-level representations always win the race, but there is a problem in dealing with the fact that letter search is delayed even when the displays are unpronounceable nonwords, and that other types of data indicate that pronounceable nonwords also are handled holistically. The problem concerns the question of what is being accessed by the displayed information.

The model that seems most consistent with both common sense and currently available data is the hierarchical model proposed by Johnston and McClelland (1980; see also Johnston, 1981). The essence of their model is that displays first activate position-specific feature detectors, which in turn activate position-specific letter detectors and inhibit letter detectors inconsistent with those fea- tures. The activated letter detectors then activate word detectors with which they are positionally consistent and actively inhibit inconsistent word detectors. Finally, the activation level of detectors is assumed to decay rather quickly, and it is further assumed that the encoding of an activated item into a form that can be subjected to cognitive operations (e.g., identification of detection) requires very slow-acting central attentional processes.

This model can handle the current data nicely if it is assumed that the assignment of cognitive codes to activated detectors either cannot occur until after word-level detectors are activated, or occurs in the reverse order of the original activation. In either case, there is a word advantage stemming from the fact that it is more likely that word detectors still are in a state of activation when the subject tries to use them than is the case for letter detectors. That is, there always is a longer time span between the initial activation of a letter detector and its ultimate use than is the case for word detectors.

One issue that remains open within this view is why subjects cannot begin the process of cognitive encoding for a letter the moment the detector is activated and before the word-level detectors are activated. There does not seem to be anything inherent to the model that precludes such immediate component-level encoding. In addition, the precise meaning of detector seems a bit open to question. For example, what does it mean to say that a letter or word detector is active but that an appropriate representation of the item is not encoded? That is, what is the distinction between an active detector for an item and an encoding? Alternatively, what is the nature of the step intervening between the activation of a word detector and the accessing of the appropriate lexical entry, and why are they not one and the same?

One possible way of dealing with this issue would be to make use of Fodor's notion of modularity (Fodor, 1983). A module might be viewed as a computational system which is completely encapsulated, both to the extent that its internal steps are not subject to feedback, and to the extent that the levels of representation from succeeding computational steps within the module are not available to immediate conscious experience. That is, only the encodings that represent inputs to modules, and the representations that constitute the output of a module, are available to attentionally driven cognitive processes, and the computational steps and ensuing representations that mediate this input-to-output transformation are not directly available to experience or volitional control.

A view such as this, added to the general model proposed by Johnston and McClelland (1980), could cope with most, if not all, of the available data. Also, this view offers a theoretical motivation for Johnston and McClelland's argument that the use of tasks that ask subjects to identify displays may not be a good approach for studying the way those items are processed. That is, because the intervening steps and levels of representation are not immediately available, any such data based on total time of processing is heavily contaminated by events that oc- 
cur after processing is complete, when subjects are attempting to retrieve (or infer) the information needed to deal with the task they are asked to perform. That data provides information about what happens after processing is finished, and not about the processing itself.

In conclusion, then, the current data seem to indicate that the word-priority effect cannot be explained in terms of truncated processing. The effect was obtained under conditions in which such processing could not occur, and it was not obtained under conditions in which subjects were able to truncate processing. The critical determiner of whether the effect was obtained seemed to be whether the subjects had an available unitary encoding that they could use to represent the display in memory.

Finally, with regard to the concept of holistic processing, it was noted that these data are consistent with any model that assumes that cognitive encodings of patternlevel representations are available before cognitive encodings of component-level representations, and they do not require the further assumption that there is no initial processing of component-level information.

\section{REFERENCES}

Chambers, S. M., \& Forster, K. I. (1975). Evidence for lexical access in a simultaneous matching task. Memory \& Cognition, 3 549-559.

Fodor, J. A. (1983). The modularity of mind. Cambridge, MA: MIT Press.

Gibson, E. J., \& Levin, H. (1976). The psychology of reading. Cambridge, MA: MIT Press.

Henderson, L. (1975). Do words conceal their component letters? A critique of Johnson (1975) on the visual perception of words. Journal of Verbal Learning \& Verbal Behavior, 14, 648-650.

JoHNSON, N. F. (1975). On the function of letters in word identification: Some data and a preliminary model. Journal of Verbal Learning \& Verbal Behavior, 14, 17-29.

Johnson, N. F. (1977). A pattern-unit model of word identification In D. LaBerge \& S. J. Samuels (Eds.), Basic processes in reading: Perception and comprehension. Hillsdale, NJ: Erlbaum.

JOHNSON, N. F. (1981). Integration processes in word recognition. In O. J. L. Tzeng \& H. Singer (Eds.), Perception of print: Reading research in experimental psychology. Hillsdale, $\mathrm{NJ}$ : Erlbaum.

JOHNSON, N. F., \& MARMUREK, H. H. C. (1978). Identification of words and letters within words. American Journal of Psychology, 91, 401-415.
Johnston, J. C. (1981). Understanding word perception: Clues from studying the word-superiority effect. In O. J. L. Tzeng \& H. Singer (Eds.), Perception of print: Reading research in experimental psychology. Hillsdale, NJ: Erlbaum.

Johnston, J. C., \& MCClelland, J. L. (1980). Experimental tests of a model of word identification. Journal of Verbal Learning \& Verbal Behavior, 19, 503-524

KrUeger, L. E. (1970). Effect of bracketing lines on speed of "same""different" judgments of two adjacent letters. Joumal of Experimental Psychology, 84, 324-330.

Marmurek, H. H. C. (1977). Processing letters in words at different levels. Memory \& Cognition, 5, 67-72.

Massaro, D., \& KLITZKE, D. (1977). Letters are functional in word identification. Memory \& Cognition, 5, 292-298.

Sloboda. J. A. (1976). Decision times for word and letter search: A wholistic word identification model examined. Journal of Verbal Learning \& Verbal Behavior, 15, 93-101.

SLOBODA, J. A. (1977). The locus of the word-priority effect in a targetdetection task. Memory \& Cognition, 5, 371-376.

THORNDIKE, E. L., \& LoRge, I. (1944). The teacher's word book of 30,000 words. New York: Teachers College, Columbia University, Bureau of Publications.

YAwORSKY, K. B., \& Johnson, N. F. (1981). Generation of visual representations. Journal of Experimental Psychology: Human Perception \& Performance, 7, 978-984.

\section{NOTES}

1. In an unpublished experiment by the senior author that employed word displays in the context of this specific paradigm, the subjects were asked, at the completion of the experimental session, whether they could report the number of overlapping letters between targets and foils. When the displays were five-letter words, subjects who experienced a targetto-foil similarity relationship of either zero, one, or two overlapping letters indicated that they did not know whether targets and foils shared letters. On the other hand, about half the subjects could describe the relationship when targets and foils shared three letters, and virtually every subject could describe the relationship when there were four overlapping letters.

2. It should be noted that the critical assumption is that rule-conforming arrays can be assigned a unitary encoding, and in these experiments, orthographic regularity and pronounceability are being used as an index of whether the display can be unitized in that manner. However, the use of those indices should not be construed as implying anything regarding the substantive characteristics of the code. In particular, it is not assumed that the code has either acoustic or phonetic properties, although such properties are not inconsistent with the pattern-unit model.

(Manuscript received January 14, 1985; revision accepted for publication August 9, 1985.) 